\title{
Políticas del don y del riesgo, en clave «nano»
}

\author{
ANALÍA ISABEL GERBAUDO Universidad Nacional del Litoral - CONICET, Argentina \\ agerbaudo@fhuc.unl.edu.ar/analia.gerbaudo@conicet.gov.ar
}

\section{Resumen}

Este artículo examina algunas de las derivas de la «ética» de la «responsabilidad» tal como se delinea en la escritura de Jacques Derrida. Hacemos foco, por un lado, en el texto Who comes after the subjetct? editado por Eduardo Cadava, Peter Connor y Jean-Luc Nancy en 1991; por el otro, en la obra de dos de sus «herederas»: Avital Ronell y Anne Dufourmantelle. Interrogamos cómo estas obras revigorizan la discusión sobre la política y la ética en el presente.

Palabras clave: ética / política / Jacques Derrida / Anne Dufourmantelle / Avital Ronell

\section{Politics of the don and the risk, in order «nano»} Abstract

This article examines questions derived from the «ethics» of «responsibility» founded by Jacques Derrida. We focus on the text Who comes after the subjetct?, edited by Eduardo Cadava, Peter Connor y Jean-Luc Nancy in 1991, and on the work of two of his "heiresses»: Avital Ronell y Anne Dufourmantelle. We ask how these works reinvigorate the discussion of the politics and the ethics in the present.

Key words: ethics / politics / Jacques Derrida / Anne Dufourmantelle / Avital Ronell

Recibido: 20/02/2019. Aceptado: 06/04/2019

Para citar este artículo: Gerbaudo, Analía Isabel (2019). Políticas del don y del riesgo, en clave «nano». El taco en la brea, 9 (diciembre-mayo), 86-102. Santa Fe, Argentina: UNL. DOI: 10.14409/tb.v1ig.8192 
¿Qué sentido tiene preguntar, cuestionar, cuando el destinatario de la pregunta no se dispone a aceptarla? Mirta Segoviano, Prólogo a Jacques Derrida y Anne Dufourmantelle, La hospitalidad

Mi «lugar-propio» también estaba constituido por mi línea telefónica (gracias a la cual puedo entregar mi tiempo, mi palabra, mi amistad, mi amor, mi amparo a quien quiero).

Jacques Derrida, La hospitalidad

I like to stay away from the dominant trends and approved protocols for reading politics.

Avital Ronell, Loser sons. Politics and authority

\section{¿Un «elogio del riesgo», entre géneros y disciplinas?}

Este texto vuelve sobre la pregunta planteada en 1991 desde el célebre volumen Who comes after the subjetct? editado por Eduardo Cadava, Peter Connor y Jean-Luc Nancy. De las colaboraciones allí recogidas, se retoma especialmente una, la de Jacques Derrida. No para examinar sus planteos (al menos no en un primer momento) sino para seguirlos a la luz de reflexiones actuales de algunos de sus innumerables «herederos», es decir, de quienes recrearon sus formulaciones para hacer con ellas «otra cosa», para llevarlas «a otra parte» (Derrida 2001a, 2001b).

De este indeterminado e inabarcable colectivo elijo a dos: Avital Ronell y Anne Dufourmantelle. Esta elección obedece a una razón heurística: sus análisis, recortados desde países, lenguas e inscripciones institucionales diferentes, atienden a la configuración de «las subjetividades poshumanistas a la luz del escenario contemporáneo»' con una particular posición, en cada caso, respecto de cómo opera tanto lo que se hace como lo que se dice (que, se sabe, también es parte de lo que se hace) desde la universidad y otras esferas de intervención intelectual. El trabajo entrelaza reflexiones más o menos estabilizadas junto con otras de carácter reciente provenientes de disciplinas y géneros en zonas de borde: el psicoanálisis, la filosofía, la teoría y la crítica literarias se intersectan en textos que «solicitan» ${ }^{2}$ tanto lo apropiado como «lo propio» ${ }^{3}$ de los protocolos académicos. Se recurre, como se verá, a entrevistas, intercambios radiales, auto-bio-grafías y cuadernos de apuntes derivados de clases y de sesiones de terapia, entre otras experiencias. Se trata, en todo caso, de un movimiento impulsado por Derrida vía su texto «"Il faut bien manger": ou le calcul du sujet» (1989a), traducido al inglés por Peter Connor y Avital Ronell como "Eating Well”, or the Calculation of the Subjetct: An Interview with Jacques Derrida» (1991a). Como aclaran Cadava y Connor en el «Prefacio» a Who comes after the subjetct?, la pregunta que se inscribe como título del volumen que coordinan se deriva de una iniciativa de Ermanno Bencivenga, editor responsable junto a Enrico Forni de la revista Topoi: su idea de organizar un dossier alrededor de «un aspecto importante de la actividad filosófica contemporánea en Francia» (Cadava y Connor:vii) termina en la edición de un número dedicado a la pregunta «Who Comes after the Subjetc» coordinado por Nancy en 1988. Como Derrida no había alcanzado a concluir su texto para dicho número, se le propone reemplazarlo por una entrevista conducida por Nancy que, de todos modos, no se llega a traducir completa al inglés al momento de la publicación. La entrevista entera se publica en francés el año siguiente, junto con el resto de los artículos que había reunido Nancy, en Cahiers Confrontation, entonces bajo la dirección de René Major. Más allá de su reedición en francés en Points de suspension. Entretiens (1992), un libro al cuidado de Elisabeth Weber que reúne una selección de entrevistas dadas por Derrida, y de su reedición en inglés en este volumen a cargo de 
Cadava, Connor y Nancy que incorpora además textos sobre la cuestión anunciada en el título a cargo de Sylviane Agacinski, Emmanuel Levinas, Sarah Kofman y Luce Irigaray (cf. Cadava y Connor), interesa poner de relieve la valoración de estos géneros académicos situados en zona de borde que revelan la importancia de la conversación y la escucha del otro en la construcción de un pensamiento: es en respuesta a otro como quien habla o escribe hace lugar a derroteros generados por una pregunta imprevisible. En la modulación de la respuesta, Derrida funda una «ética» ${ }^{4}$ sostenida en la «responsabilidad». ${ }^{5}$

\section{¿ «Goza tu síntoma!»?}

En febrero de 2012 sale a la venta un pequeño libro editado por el sello Odile Jacob. Se trata de Le sel de la vie. Lettre à un ami de Françoise Héritier. Su primera frase caracteriza con exactitud el efecto que dicho texto causó, al menos en quien escribe este artículo: «Le texte qui suit risque de surprendre ceux qui me connaissent par mes écrits anthropologiques» (Héritier, 2012a:7). Efectivamente, esa publicación sorprende a esos lectores a los que Héritier alude, además de ser la primera de una serie que continuaría hasta donde se lo permitió la vida (Héritier 2017). Se trata de una serie relativamente al margen de los estudios que, hasta entonces, formaban parte de su obra: conocida por Masculin/ Féminin I. La pensé de la différence (1996) y Masculin/ Féminin II. Dissoudre la hiérarchie (2002), entre otros textos hoy legibles en términos de combates ${ }^{6}$ sexogenéricos (Gasparri) ${ }^{7}$ — combates en los que ha adoptado posiciones verdaderamente controversiales (cf. Eribon)—, Héritier pone a circular un texto que, más allá de sus declaraciones, no evidencia, en principio, una preocupación teórica central. Si creemos en sus cuentos, ${ }^{8}$ diríamos, junto a ella, que se trata de «une fantaisie» (2012a:7), de «une flânerie» (2012b). Si atendemos a nuestra lectura diríamos que, efectivamente, se trata de un escrito que, más allá de cierta tendencia excesivamente propositiva (me apuro en aclararlo: Héritier no se inscribe en una línea de reflexión marcada por la producción de Derrida) y de su ciertamente cuestionable «valor literario» (término ambiguo sobre el que sería distractivo volver aquí), interesa por la preocupación que expresa.

Héritier reacciona sintomáticamente ${ }^{9}$ vía la palabra a una acción generada por otra palabra: se trata de una tarjeta postal que le había enviado quien fuera su médico por más de treinta años, «Monsieur Piette» (7). Esa tarjeta tenía un comienzo, para Héritier, inquietante: «Monsieur Piette» había escrito «une semaine “volée” de vacances en Écosse» (Héritier 2012a:7). Es esa expresión la que desata, en principio, una referencia a la abnegación y a la entrega con la que «Monsieur Piette» hacía su trabajo; luego interroga dicha referencia desde un tópico que atraviesa la producción de Derrida, la de Dufourmantelle y, en menor medida, la de Ronell: «el don» del tiempo. Recorto ese pasaje:

Et voilà que ce terme: une semaine «volée» me saute littéralement à la figure. Qui vole quoi? Vole-t-il donc un peu de répit à un monde auquel il devrait tout ou au contraire ne se laisse-t-il pas déposséder de sa vie par cet entourage dévorant, ce travail obsédant, ces responsabilités multiples accablantes? (Héritier 2012a:8)

Apenas unos años después, Anne Dufourmantelle asiste a varios programas de la radio France Culture (Dufourmantelle 2014, 2016) para tratar un tema entonces candente desatado por la serie de atentados que se producen prácticamente en paralelo con la publicación de su libro Éloge du risque (2011). La escucha psicoanalítica de Dufourmantelle le permite elaborar una serie de conjeturas y de preguntas que traslada con precisión e inteligencia de casos individuales al tejido social. 
Sus planteos giran alrededor de la constitución de la subjetividad enfatizando el resto o margen que le queda al sujeto para la decisión «responsable». Término que lleva la marca de la posición derrideana cuyos ecos se advierten en al menos uno de los dos epígrafes de su, entonces, reciente libro: «L'instant de la décision est une folie», afirma indirectamente Dufourmantelle trayendo el eco de la palabra de Soren Kierkegaard (2011:5). Esta insistencia, tanto en Derrida como en Dufourmantelle, desliza al menos dos tensiones: por un lado, la que se produce entre lo que la ley (arbitraria como todo texto y, por lo tanto, desconstruible: «es una locura la ley», repite Derrida en «La loi du genre» —1980c:266一) le hace a los sujetos mientras hace a los sujetos, mientras regula sus márgenes de acción (un tema que Derrida trata en deuda con Franz Kafka —Derrida 1985 - y con Walter Benjamin —Derrida 1994-). Por otro lado, se inscribe la tensión que se produce al poner de relieve la relación entre visión y ceguera (cf. Derrida 1990a, 1998a, 2000) en toda decisión responsable: se da respuesta a algo o a alguien en una situación de encrucijada, sin tener pleno dominio de los engranajes de la maquinaria en la que esa respuesta se inserta. ${ }^{10}$

Es en esa línea que Dufourmantelle interroga el régimen actual de "servitude volontaire»" (2011) de la que los sujetos participan desde un enclave que reactualiza la tensión derrideana entre el don y la deuda (ambos, a la vez, endeudados con Jan Patocka). Sus intervenciones cuestionan la administración contemporánea del tiempo: en una cultura que ha convertido al trabajo en «valor absoluto», dar la vida al trabajo, «¿es perderla o ganarla?» (Dufourmantelle 2014). «Esconder la cabeza debajo del agua para no perder las ventajas obtenidas», «no hacer nada que ponga en peligro el status quo» son para Dufourmantelle respuestas individuales a una sociedad que hace de la «eficacia» y de la «eficiencia» económica sus valores dominantes (1997:74-76). «Devuélvalos reparados» es la exigencia que subyace a la solución farmacológica, en alza, vía la psiquiatría: si el síntoma es la reacción que pone un freno, no sólo a la aceleración vertiginosa del tiempo sino también a la demanda creciente de estar disponible las 24 horas de cada uno de los 365 días del año (Rodríguez Freire), la medicación, lejos de interrogar lo que provoca el síntoma, por lo general, salvo patologías que realmente la necesiten, «normaliza» en el sentido foucaultiano del término, es decir, vuelve el cuerpo dócil a la maquinaria en la que se pretende insertarlo sin resistencias (Foucault). ${ }^{12}$

No obstante, Dufourmantelle va un poco más allá: «uno de los lugares más fuertemente atacados es, efectivamente, el lazo, la relación con el otro» (2014). Este comentario se hilvana con otra obsesión ${ }^{13}$ derrideana que sus textos recrean: la atención al otro radicalmente otro, al extranjero, al desconocido por-venir y la fantasía de la hospitalidad.

«Si en hebreo, "fabricar tiempo" es equivalente a "invitar", ¿cuál es esta extraña inteligencia de la lengua que certifica que para producir tiempo es preciso ser dos, o más bien es preciso que exista lo otro, una efracción de lo otro?» (Dufourmantelle 1997:78). En 1997, Dufourmantelle escribía esto, a modo de respuesta a su escucha de las clases impartidas por Derrida en su seminario sobre la hospitalidad y la hostilidad. De aquellas sesiones, Derrida y Dufourmantelle eligen dos para publicarlas en un libro también de inclasificable estatuto genérico: la clase cuarta, «Pregunta de extranjero: venida del extranjero» y la quinta, «No existe hospitalidad» se combinan con la «Invitación» realizada por Dufourmantelle bajo un título que enreda preguntas y respuestas en un bucle sin fin: Anne Dufourmantelle invite Jacques Derrida a répondre De l'hospitalité (1997). Un libro traducido al español por Mirta Segoviano para Ediciones de la Flor como La hospitalidad (2000).

«Pas de hospitalité» es una expresión ambigua inserta en un texto que hace serie con otros en los que Derrida insiste en el miedo a lo por-venir, a lo que desacomoda nuestros esquemas 
y nuestros órdenes, al «acontecimiento» (Derrida 1993, 1998b, 2003a, 2003b) o, en términos de Dufourmantelle, al «riesgo»: «No existe hospitalidad», «Basta de hospitalidad», «Nada de hospitalidad» son las variantes con las que juega la traducción al español propuesta por Segoviano para un texto que resalta el límite que la norma vía la moral y el derecho, impone a una ética y a una justicia situadas «más allá» de estas fronteras. Como planteará más tarde para «la universidad» (Derrida 2001c), la «hospitalidad» exige un carácter de «sin condición» (cf. Chun) que roza lo imposible dadas las constricciones que enfrenta: «todo ocurre como si las leyes de la hospitalidad consistiesen, al marcar límites, poderes, derechos y deberes, en desafiar y en transgredir la ley de la hospitalidad, la que ordenaría ofrecer al recién llegado una acogida sin condición» (Derrida 1997:81).

"Le risque est beau», resalta Dufourmantelle vía la palabra de Platón que inserta como otro de los epígrafes a su «elogio del riesgo» (2011). Sus intervenciones amontonan interrogantes que dejan entrever grietas ${ }^{14}$ para «desobediencias», intersticios que permiten abrigar la fantasía de inventar la propia vida. A la procastinación expresada en la idea de que «la verdadera vida comienza mañana (un mañana de diez minutos, diez años o de toda una vida)» y a la justificación para la inacción sostenida en que, «ante la dificultad para cambiar todo, no vale la pena cambiar nada», Dufourmantelle opone el resquicio para lo inédito: a pesar de la «toxicidad fenomenal» que halla en la ideología del control y de la seguridad tendientes a reducir la vida y al sujeto a su sola «supervivencia individual» (2014), neurótica y temerosamente programada y programable, traduce a clave psicoanalítica la potencia del acontecimiento y de lo insospechado que solicitan el sentido de términos como «perder» $\mathrm{y}$ "ganar» cuando se imprimen sobre palabras como «vida» $\mathrm{y}$ «tiempo»: ¿Qué sería arriesgar la vida? ¿Y qué perder el tiempo? ¿Y si cuando se cree perder el tiempo o la vida en verdad se los gana? ¿En términos de qué parámetros se gana o se pierde el tiempo? ¿Cuándo se dona? Fiel porque infiel a Derrida (fiel en sus preguntas, infiel en su tendencia a sugerir líneas de orientación posibles), en sus textos se escucha el eco de sus consideraciones en Donner le temps:

El don, si lo hay, será siempre algo sin borde. ¿Qué quiere decir aquí «sin»? Un don que no desborda, un don que se dejase contener en una determinación y limitar por la indivisibilidad de un trazo identificable no sería un don. En cuanto se delimita, el don es víctima del cálculo y de la medida, del dominio y de la métrica, de la salvaguarda del control. (Derrida, 1991b:93)

Fiel a Derrida, Avital Ronell sostiene la potencia política de las intervenciones tramadas desde el espacio académico: como Derrida, apuesta a lo que pueden las artes y, en particular, la literatura. Así como Derrida funda en la literatura su programa-no-programático (cf. Gerbaudo 2007), Ronell exacerba las «contaminaciones» disciplinares y de géneros en una producción marcada por el control y la exhibición desaforada de las estrategias que moviliza: «Soy una universitaria que continúa firmemente creyendo en la capacidad de escándalo que sólo la literatura y la poesía detentan» (2011:289), le responde a Vincent Kaufmann en La culpa la tiene Mallarmé..., un texto que acusa recibo de las defenestraciones a algunas de las líneas de la teoría perpetradas por una serie de firmas entre las que se destacan Antoine Compagnon y Tzvetan Todorov. Inteligente y cauto, Kaufmann discute a través de la voz de otros: además de la reconstrucción de la polémica, recoge un conjunto de entrevistas a varias de las rock stars de la teoría para componer un panorama verdaderamente complejo. Entre esas figuras rutilantes está Ronell. El potencial de «escándalo» que Ronell encuentra en la literatura se traslada a la teoría que se deriva de ella (2011:286): siguiendo 
las lecciones derrideanas que a su vez reinventa, examina aspectos minúsculos que le permiten esbozar conjeturas sobre grandes tópicos y problemas de la cultura (1992, 2002, 2012).

$\mathrm{Al}$ respecto, durante las entrevistas con Anne Dufourmantelle publicadas en francés con el título American philo (2006), se destacan tres cuestiones: 1) la descripción de la incomodidad que la llamada French Theory (un movimiento que reúne a pensadores tan diferentes como Gilles Deleuze, Derrida, Michel Foucault, Julia Kristeva, Jacques Lacan, Roland Barthes, Georges Bataille, Jean-Luc Nancy, Philippe Lacoue-Labarthe, Pierre Bourdieu, Alain Badiou, Jacques Rancière, Étienne Balibar, Jean-François Lyotard, Hélène Cixous, René Major, etc. —Ronell 2006:184一) provoca en la academia norteamericana; 2) la caracterización de los «desplazamientos» (181) de lo enquistado en la universidad estadounidense vía la desconstrucción, ese movimiento «escandaloso» (131) impulsado por Derrida; 3 ) la valoración del «riesgo», del «pensamiento que se expone» sabiendo que pagará por ello un precio (258). Ronell rememora la desestimación de su tema inicial de doctorado (un trabajo sobre Benjamin, entonces un «desconocido» en la universidad norteamericana -17-) junto a otros episodios de rechazo: la conmoción que provoca su intento de abordar la pedofilia en Goethe como posible tópico de investigación (21); más tarde, sus traducciones, clases y, fundamentalmente, su singular apropiación de Derrida en sus ensayos críticos (cf. Ronell 1992, 2002, 2012). Esta selección de episodios intenta mostrar la escala «nano» en la que Ronell interviene (2006:123, 252; 2008; 2011:288; 2012:xiii). Es justamente durante un coloquio que llevaba por título «Derrida político»" ${ }^{15}$ cuando desarrolla su provocativo concepto de "nanointervención» que alude a operaciones ceñidas a la "pequeña tarea», opuestas a «lo espectacular» (2011:289) y tendientes a «interrumpir» los flujos naturalizados en la lectura de la cultura. Se trata de «cuestionamientos cruciales» inspirados en Kafka que, a su vez, inspira a Derrida. Mediante un «bucle extraño» (Hofstadter), mientras habla de los otros, habla de su propio trabajo: Ronell lee la desconstrucción con la exigencia de una performatividad limitada y encuadra su activismo dentro de la «nano-política» (2008) que, lejos de su reducción, revela la responsabilidad con que encara la tarea: si el efecto logrado es una suspensión del tipo de las que ensaya, los objetivos estarían cumplidos. La «suspensión» como forma particular de la «sintaxis de la acción» (2006:56) atrae su atención tanto como la de Dufourmantelle y, como ya vimos, la de Derrida.

Cabe destacar que las entrevistas recogidas en American philo exponen la confrontación de hipótesis tanto en las preguntas de la entrevistadora como en las respuestas de la entrevistada. Por ejemplo, sobre el «hilo» de lectura (Derrida 1972b) seleccionado para esta presentación, Dufourmantelle cuenta una historia que alguna vez le relataron en India: la escena inicial de su cuento presenta a un maestro planteándole a uno de sus discípulos un enigma. El relato se tensa por diferentes escenas que repiten la misma circunstancia: cada vez que el discípulo cree hallar la solución al enigma, el maestro lo pone ante un nuevo desafío. Esa tensión dura hasta la muerte del maestro. El discípulo llega dos días después de su fallecimiento. Frente a la situación, expresa su tristeza por no haber podido exponerle la nueva solución que había imaginado. Un discípulo lo escucha y le responde: «Tal vez has encontrado la solución, de cualquier modo. Era el camino: el camino que tú hacías para venir a verlo, el trayecto que no veías porque estabas demasiado ocupado en llegar a tiempo» (Dufourmantelle 2006:59). Es a partir de este relato que Ronell interroga cuándo y cómo se produce pensamiento. Si bien no da una solución formularia, muestra su alerta respecto del «deseo de terminar lo más rápido posible, la impaciencia, el frenesí de concluir» (Ronell 2006:57), el exceso de trabajo convertido en una suerte de «droga» cuyo con- 
sumo es incentivado por las mutaciones tecnológicas (97) y la cultura del «test» (61). Por su parte, Dufourmantelle bosqueja algunas ideas sobre las que volverá en otras intervenciones y que centra en dos hilos que anuda: mientras que toma distancia respecto del modo en que «nuestra época estigmatiza las reacciones intempestivas» (se trata, afirma, de producir un «cuerpo no excitado, tranquilo, dócil» - 55-), por el otro, lee en algunos síntomas como la depresión, formas de «refugio» contra los «elementos mortíferos de la cultura» (55).

Cuerpos enfermos, cuerpos-desecho, cuerpos narcotizados. En cualquier caso, cuerpos no indiferentes a las tensiones que los atraviesan. Cuerpos que no participan de esa forma del cinismo que, más allá de algunos matices de su obra que no compartimos (en especial cuando habla de la desconstrucción) y sobre los que no vamos a desviarnos aquí, expone con agudeza Slavoj Zizek en ¡Goza tu síntoma! Hacia 1992, Zizek opone la distancia cínica privada que observaba en Europa Oriental respecto de los rituales públicos de obediencia al cinismo que observa en Occidente: «públicamente simulamos ser libres mientras que en privado obedecemos» (10). Y agrega: «en ambos casos, somos víctimas de la autoridad precisamente cuando creemos que la hemos embaucado: la distancia cínica está vacía, nuestro verdadero lugar se encuentra en el ritual de la obediencia» (11). Dicho en los términos de Kurt Vonnegut que Zizek trae vía Madre Noche: “Somos lo que simulamos ser, de modo que debemos tener cuidado con lo que simulamos ser"» (11).

\section{¿Un voluntarismo no voluntarista, un programa no programático?}

Menos prescriptivo que Dufourmantelle y menos denuncialista que Ronell (y, cabe remarcarlo, en las antípodas de cualquier gesto que roce alguna moral, por más que se trate de un escrito auto-bio-gráfico como el Héritier), Derrida tiene la sutileza para plantear un problema dejando como respuesta una pregunta, aun cuando escribe a partir de preguntas de otro. Así, en el texto de 1991 que inspiró estas páginas, esboza tres o cuatro interrogantes centrales para los debates que vendrán: entre ellos, los que aquí seguimos, entre los muchos otros que deslizan sus incontables herederos, incluidos Zizek (muy a su pesar), y Nancy quien, como Dufourmantelle cuando conversa con Ronell, revela sus hipótesis junto a sus preguntas en la ya mencionada entrevista.

Nancy propone, en su carta de invitación, dos puntos a considerar en el supuesto artículo que iba a escribir Derrida. El primero, «Qui vient après le sujet?» (Nancy en Derrida 1989a:269); el segundo, «Un discours, répandu dans une époque récente, conclut a sa simple liquidation» (269).

Derrida empieza por subrayar que la primera cuestión acarrea el «supuesto» de que algo como el sujeto pueda identificarse mientras que la segunda, abriga la «ilusión» de que algo así como la «liquidación» pudiera perpetrarse (Derrida 1989a:270). Respecto de la primera, aclara: «Je n'accepterais pas d'entrer dans une discussion au cours de laquelle on supposerait savoir ce qu'est le sujet» (273). Respecto de la segunda, advierte que si trae ciertos nombres propios (Lacan, Althusser, Foucault — todos ellos, remarca, en deuda con Freud, Marx y Nietzsche—) no es sino para «responder» a cierta doxa que se apresuraría en decir «todos estos filósofos creen haber dejado el sujeto detrás de ellos» (272).

Esta insistencia de Derrida sobre los puntos que Nancy plantea para la discusión responde a haber encontrado en ellos el eco «de un discurso de opinión» (273) que cree oportuno solicitar. Y su modo de hacerlo es interrogar su «definición» desde desarrollos teóricos con derivas éticas y políticas que están en textos previos a los que envía. Así, su respuesta no tendrá sino la forma de una pregunta: «si hay un sujeto", ¿sería acaso aquel que responde "si, sí" antes de poder formular 
una pregunta?», ¿sería aquel que ensaya decisiones responsables «aunque sin autonomía»? (275). Si bien en nota al pie se envía a Éperons, Parages, Préjugés, Ulysse Gramphone, De l’esprit, «Nombre de oui», también se podría enviar a Glas, La carte postal y en especial a Signéponge debido al resaltado que viene a continuación y que dejará una marca notable en el texto que muchos años después Nancy escribirá en su homenaje: «Plus d'un Derrida, plus d'un Jacques ou d'un Jackie, plus d'un J., plus d'un D., plus d'un trait» (Nancy 2007:95). Dice Derrida: «Le rapport à soi ne peut être, dans cette situation, que de différance, c'est-à-dire d'altérité ou de trace» (1989a:275). A este señalamiento, con sus respectivos envíos, agrega uno más: el de «una cierta responsabilidad» (276) constituida por la respuesta al otro: «La singularité du "qui" n'est pas l'individualité d'une chose identique à elle-même, ce n'est pas un atome. Elle se disloque ou se divise en se rassemblant pour répondre à l'autre, dont l'appel précède en quelque sorte sa propre identification» (276). Derrida habla de dehiscencia, dislocación intrínseca, différance, destinerrancia, etc., mientras se pregunta tanto si es posible seguir hablando de sujeto tomando tales características como si cabe prohibir-se hablar de sujeto apelando a tales términos (280). «No esperen de mí una respuesta con la dimensión de una fórmula», advierte mientras agrega: «esto no depende de un decreto especulativo, menos aún de una opinión. Tal vez no solamente de la discursividad filosófica» (281). No obstante, si bien no se halla en su larga conversación una respuesta, cabe subrayar un pasaje en el que remarca otra de sus grandes líneas que desarrollará en los años que vendrán. Plantea aquí una clave que explica sus derivas sobre el cuestionamiento de «lo propio del hombre» por contraste con el animal:

Pourquoi ai-je rarement parlé de «sujet» ou de «subjectivité», mais seulement, ici ou là, d' «effet de subjectivité»? Parce que le discours sur le sujet, la même où il reconnait la différence, l'inadéquation, la déhiscence dans l'auto-affection, etc., continue à lier la subjectivité a l'homme. Même s'il reconnait que l'«animal» est capable d'auto-affection (etc.). (283)

Incómodo con toda conceptualización ${ }^{16}$ dado su carácter necesariamente reductor, Derrida insiste en la condición problemática del concepto de «sujeto» debido a su necesaria asociación con principios de «cálculo» como los que rigen el derecho, la moral y la democracia a los que opondrá una justicia, una ética y una democracia por-venir regidas por los derroteros del don, la hospitalidad y la respuesta al otro-radicalmente-otro, probablemente monstruoso, de venida inesperada, fuera de cálculo y de todo programa. Para Derrida no hay «responsabilidad ni decisión ético-política que no deba atravesar los pasajes de lo incalculable y de lo indecidible» (287). Es para contribuir a generar otro estado de las cosas que vuelve sobre la maquinaria conceptual que había permitido hablar de sujeto, derecho, moral y democracia tal como se hacía hasta entonces. Derrida revisa la autoridad del estar-presente alrededor del cual se estructuró el derecho, la política y la moral: presencia a sí, identidad, propiedad, consciencia, libertad, voluntad, intencionalidad, humanidad. No se trata, aclara, de generar un discurso sobre las mismas cosas sino de solicitar la maquinaria conceptual que las estabiliza: porque cree que su «análisis» es «eso y algo más», porque cree que su «análisis» es traducción de una transformación en curso, puede leer el «nerviosismo» que le genera a quienes quieren mantener estos asuntos al abrigo de todo cuestionamiento, es decir, de todo pensamiento, de toda desconstrucción del carno-falogocentrismo que se convertirá, casi hasta el final de sus días, en su obsesión. En un esquema dominante regido por la concepción-apropiación-asimilación del otro, Derrida vuelve sobre la metonimia del «bien manger». «Puisqu’il 
faut bien manger (...), comment faut-il bien manger? Et qu'est-ce que cela implique? Qu'est-ce que manger?» (296), pregunta. Un poco más adelante trae otra metonimia: Auschwitz. Derrida solicita qué nombra el nombre de "Auschwitz»: «Quel est ici le référent? Fait-on usage métonymique de ce nom propre? Si oui, qu'est-ce que le règle?» (301).

Como toda respuesta, Derrida deja la ambigua y exigente acepción de «responsabilidad», clave de su ética: «Repito: la responsabilidad es excesiva o no es tal. Una responsabilidad limitada, medida, calculable, racionalmente distribuible, es el devenir derecho de la moral: es tal vez el sueño de todas las buenas conciencias, en el mejor de los casos; de los pequeños o grandes inquisidores, en el peor» (301).

Estas cuestiones atraviesan buena parte de los textos que escribe desde entonces. Sorprende cierto virage «comunicacional» en sus últimos trabajos sobre este punto. Tal vez se trate de un intento de hacer llegar a un público expandido sus formulaciones sobre estos problemas. Hago referencia aquí, entre otros, a su diálogo con Élisabeth Roudinesco (2001a), a las entrevistas que cede para las muy cuidadas ediciones de «A voix nue» emitidas por la radio France Culture (2001b), a sus discusiones con Giovanna Borradori a propósito de los sucesos del llamado «11 S» (2003a) y a sus clases, tanto las recogidas junto a Anne Dufourmantelle (1997) tomadas de su seminario sobre la hospitalidad y la hostilidad como las póstumas y últimas sobre «la bestia y el soberano» $(2008,2010)$.

Derrida ya lo había advertido entonces: no alcanza con un llamado ni con una agenda ni con un programa (1989a). Se trata, en todo caso, de una discursividad destinerrante que bosqueja y actúa una ética resuelta por el modo en que damos respuesta, lejos del voluntarismo o, en todo caso, más cerca de un voluntarismo no voluntarista o de una noción de voluntad ajena al voluntarismo con algo de ceguera, es decir, con algo de la visión que se desprende de estados mayoritarios de ceguera y secreto, incluso para sí. Visión, ceguera, secreto: metáforas que son algo más que meras figuras de la compleja y exigente política que se desprende de su obra, de toda su obra, estimulante porque im-posible, siempre entonces in-completa, trunca, in-abarcable para todo aquel que ose abordarla. ${ }^{17}$

\section{Notas}

1 Retomo parte del texto escrito por Raúl Antelo y Susana Scramim a los fines de difundir el coloquio que organizaron el 4 de setiembre de 2018 para discutir, junto a Carlos Capela (UFSC), Jorge Wolff (UFSC), Luz Rodríguez Carranza (Universidad de Leiden) y Max Hidalgo Nácher (Universidad de Barcelona), las derivas actuales de una pregunta instalada en el campo del pensamiento occidental hace ya treinta años. Quiero agradecer muy especialmente la invitación a participar de este diálogo así como las preguntas que se plantearon en él (en especial, las de Jorge Woolf, Susana Scramim y Oscar Reymundo). Esta versión, presentada para su evaluación en este dossier coordinado por Jorge Woolf y Susana Scramim, expande mis primeras respuestas.
2 Este término se usa con el sentido derrideano de hacer temblar, desestabilizar, descalabrar, interrogar las certezas alrededor de algo.

3 La cuestión de «lo propio» tiene en Derrida matices muy variados (cf. 1972a, 1974, 1984, 1990b, 1997, 1999). En algunos textos su solicitación de «lo propio de» se lleva hacia una crítica a los esencialismos desde la «bonita insolencia» (la expresión es suya) de mostrar que, más que «lo propio de», hay «efectos de» (así como no hay «la» desconstrucción sino efectos de desconstrucción). Por ejemplo, en Dar el tiempo dirá: «No hay naturaleza, solo efectos de naturaleza: desnaturalización o naturalización» (1991b:166). En Glas el problema toma otros matices: Derrida solicita el carácter «propio» del llamado «nombre propio» mientras 
interroga los juegos anagramáticos de Jean Genet. Por un lado, Genet inscribe su nombre en los textos que firma explotando los varios sentidos que dicho término evoca: «Genêt nomme une plante à fleurs - jaunes (...genêt-a-balais, vénéneux et médicinal...), genet une espèce de cheval» (1974:48). Por otro lado, Derrida se pregunta qué hace Genet cuando convierte nombres comunes en nombres propios de sus personajes. Sin fijar una respuesta, la detención en esta estrategia vuelve sobre el poroso límite entre lo común y lo propio: «Quand Genet donne à ses personnages des noms propres, des espèces de singularités qui sont des noms communs majusculés, que fait-il? Que donne-t-il à lire sous la cicatrice visible d'une émajusculation qui menace toujours de se rouvrir? S'il appelle Mimosa, Querelle, Divine, Yeux-Verts, Culafroy, Notre-Dame-des-Fleurs, Divers, etc., arrache-t-il violemment une identité sociale, un droit de propriété absolue? Est-ce là l'opération politique la plus effective, la pratique révolutionnaire la plus signifiante?» (10-11). En Signéponge abre otra dimensión del problema: Derrida interroga cómo se construye un «nombre propio» (si tal cosa existe) a partir de la escritura de Ponge, plagada de anagramas de su propio apellido, de homónimos, de efectos «fr» (es decir, de juegos con el sonido de las primeras iniciales de su nombre), de personajes que se llaman F. P., de pasajes que remedan la tensión entre el escribir y el borrar. El título anticipa estas preguntas propiciando la interrogación respecto de cómo se construye una firma. Finalmente podría conjeturarse que es a partir de Genet y de Ponge, entre otros autores, que Derrida solicita qué es lo «propio de» la literatura (se advertirá que se trata de problemas imposibles de abordar en una nota, más allá de que era necesario señalar la complejidad que la interrogación de «lo propio de» cobra en su escritura).

4 Para una lectura convincente e incisiva de la ética y la política en Derrida a la luz de las tensiones del presente, ver Chun.

5 Derrida diferencia la «moral», asociada a la «buena conciencia», y la «ética», asociada a la «responsabilidad» (Derrida 1989b). Para Derrida hay «responsabilidad» cuando hay «experiencia», cuando se está ante una «aporía», cuando se debe afrontar el riesgo de tomar una «decisión». En esa línea se pregunta: «¿es posible una experiencia que no sea experiencia de la aporía?» (1996:34). Para Derrida la moral se mueve en un plano de acción regulado por lo «conforme al deber» y a lo actuado «por deber», por sujeción a la «ley» regulada por el «derecho» mientras que la ética supone afrontar una «decisión» que no se limita a "poner en marcha un saber determinable o determinante» o «la consecuencia de algún orden preestablecido» (37). La decisión interrumpe «la relación con cualquier determinación presentable» pero a la vez mantiene «una relación presentable con la interrupción y con lo que esta interrumpe» (37). Esta ética supone actuar desde la lógica de la différance: cuando Derrida se pregunta si es esto posible «dado que la interrupción siempre se parece a la marca de una linde fronteriza, de un umbral que no hay que traspasar» (37) trae la figura de la temporalidad que apela a «una différance en el ser consigo del presente» (37). Mientras la moral supone «la buena conciencia como mueca de una vulgaridad complaciente», «la forma segura de la conciencia de sí» (40), la ética ligada a la decisión responsable supone exponerse al «compromiso» con el «riesgo absoluto» (40): «Tal vez se podría sacar la conclusión de que la esencia de la decisión, aquello que la convertiría en el objeto de un saber temático o de un discurso teórico, debe permanecer indecidible: para que haya, si es que la hay, decisión» (96).

6 Partir de una concepción de la «academia» como espacio de combate, tal como lo han hecho sociólogos, filósofos, psicoanalistas, críticos literarios (cf. Bourdieu 1984, 2001; Carles; Ronell 2006:8-9, 42, 45, 47; Compagnon) permite realizar análisis que sortean los giros morales observables en los planteos transidos por el lamento por lo no inscripto o lo excluido de ese espacio en diferentes coyunturas socioculturales. Por otro lado, pensar a los conceptos y a los desarrollos teóricos como armas de combate y de intervención supone reconocerles un potencial político en tanto contribuirían a desarrollar acciones críticas y modificaciones de cierto estado de las cosas (cf. Rinesi 2003). Así por ejemplo, la versión en inglés del diálogo que sostienen Ronell y Dufourmantelle se traduce en 2010 como Fighting theory. Avital Ronell in conversation with Anne Dufourmantelle. Es oportuno señalar que esta posición, además, se reitera en la mayor parte de los trabajos de Bourdieu y de Ronell: sus investigaciones y análisis críticos se piensan como trabajos específicos que responden a la lucha contra algo que se quiere discutir, debatir y/o contribuir a corroer.

7 Empleamos este término siguiendo las consideraciones de Javier Gasparri en sus notas introductorias al dossier bajo su coordinación «Perspectivas sexogenéricas: literatura, artes y política»: «las perspectivas sexogenéricas se plantean como formulaciones críticas con diferentes modulaciones y puntos de vista, es decir, con configuraciones conceptuales que abrevan en diferentes teorías, tradiciones filosóficas y disciplinas. 
Las agrupamos, así, como "sexogenéricas" ya que la cuestión sexo/género posee inflexiones históricas en su propio devenir conceptual, así como distintos enfoques epistemológicos, y además, porque da cuenta del énfasis sexual ineludible, esto es, el modo en que las sexualidades no pueden pensarse sino en relación con el género, y a la inversa. Pero también, y sobre todo, alía a estas perspectivas el hecho de que se afirman en disidencia respecto del falogocentrismo, la heterosexualidad obligatoria y normativa, el heteropatriarcado hegemónico, y se proponen desmontarlos, subvertirlos, abolirlos, corroerlos, destruirlos, desobedecerlos, entre otras estrategias» (2). La precisión de Gasparri exhorta a reponer algunos de los aportes al problema. Se trata de dos textos tempranos de notable vigencia ya que siguen aportando al análisis de problemas actuales. Por un lado, «La loi du genre», ensayo en el que Derrida (1980c) esboza una noción de género crucial para desestabilizar el positivismo de las clasificaciones estables y estabilizadas a las que conducía el término: en la línea profanatoria de «Il n'y a pas de hors texte» (1967:228), casi veinte años más tarde afirmará que todo texto «participa de más de un género sin pertenecer con exclusividad a ninguno» (Derrida 1980c:237). Se trata de una de las afirmaciones más radicales del pensamiento del siglo XX: su potencia heurística está dada por los análisis a los que abre, tal como lo muestra la producción posterior, en deuda con esta noción (Butler). Por otro lado, La dominación masculina (Bourdieu 1999), si bien con hipótesis circunscriptas al espacio francés, contribuye a reflexionar respecto de lo que queda por hacer en el plano social dada la pregnancia que los habitus heteropatriarcales ejercen en las prácticas cotidianas.

8 Para el análisis de los relatos de los académicos sobre sus propias producciones, trayectoria, decisiones, etc., en términos de «cuentos», ver Gerbaudo 2018.

9 Uso el concepto de «síntoma» según la muy poco convencional acepción de Paco Vidarte. En «Derriladacan: contigüidades sintomáticas. Sobre el objeto pequeñoj@cques» enreda el conceptos de «síntoma» («lo que te cae») con el de «acontecimiento» («lo que te toca»): «Síntoma viene del griego sin-pípto [ind.: pet-], que significa, "caer juntamente", "coincidir". Designa así algo tan simple como que dos cosas vengan a caer juntas, una al lado de la otra, dos cosas coinciden, y ello puede o no suceder "propiciamente" (sin salirnos de esta familia); también lo que cae hacia adelante, lo que se precipita sintomáticamente que viene a ser lo mismo que fortuitamente. De síntoma procede también el término "asíntota", lo que se acerca mucho pero no coincide nunca. Y desecho, cadáver (ptóma), lo que cae. Y repetición.

Acontecimiento viene de tango, contigere [ind.: tag-]. Lo que acontece es lo que toca, en el sentido del tacto. Lo que te toca, por contigüidad, término éste también emparentado. Y nos toca "contingentemente", accidentalmente, azarosamente. De aquí viene asimismo "tangente", lo que toca. $\mathrm{Y}$ "contaminar", corromper tocando. Noli me tangere, dice el acontecimiento.

Acacecer y acaecimiento provienen de cadere, caer [ind.: kad-]. El acaecer cae como cae el síntoma. Acaecer quiere decir también armonizar o acompasar las voces de los que cantan. El portugués ha dado "esquecer", que es olvidar. De esta raíz proceden también "accidental”. Y “coincidir” y, de nuevo, cadáver.

Tanto en la esfera del acontecer/acaecer como en la del síntoma nos movemos en un mismo ámbito de contingencia y accidentalidad. La alusión siempre es a dos cosas que caen juntas o que se tocan, por coincidencia o contigüidad. Podríamos decir también por metonimia. Dos cosas, una al lado de la otra. Eso es un acontecimiento, lo que nos toca, lo que cae junto con nosotros y establece una relación de contigüidad metonímica, de coincidencia tangencial o asintótica (la asíntota es tangente en el infinito)» (19).

Esta osada definición se desprende de la también extravagante definición de Derrida que la motiva y que Vidarte coloca como epígrafe de su trabajo, vía su traducción: «En todos los casos, la incidencia se deja marcar en el sistema de una coincidencia, la misma que cae, bien o mal, con otra cosa, al mismo tiempo o en el mismo lugar que otra cosa. Ése es también en griego el sentido de symptôma, palabra que significa en primer lugar el hundimiento, el desplome, luego, la coincidencia, el acontecimiento fortuito, el encuentro, también el acontecimiento desafortunado y, finalmente, el síntoma como signo, por ejemplo, clínico» (Jacques Derrida, Mes chances en Vidarte). El concepto de Vidarte se deriva de una apropiación desacralizante de los aportes de Sigmund Freud (1895) y de Jacques Lacan (1975-1976) impulsada, a la vez, por las heterodoxas articulaciones derrideanas.

10 La dificultad para develar el secreto, no sólo a otros sino a uno mismo, atraviesa el pensamiento de Derrida: «Personne ne saura jamais à partir de quel secret j'écris et que je le disse n'y change rien» (1991c:175), aclara en «Circonfession». Esta dificultad de pasaje de un plano de reflexión a otro, este fracaso de todo metalenguaje, también se observa en Dufourmantelle. En Intelligence du rêve. Fantasmes, apparitions, inspiration (2012) 
revela el papel del sueño en el descubrimiento de verdades ocultas para el propio Sujeto (su dedicatoria es equivalente a un resaltado en negrita dado que se trata de un pasaje en español inserto en un libro enteramente escrito en francés: «A quien sabe lo que pueden los sueños» [2012:7]). Estas verdades que emergen durante ese estado de caída del control. No obstante, deben pasar por los filtros que el recuerdo de lo soñado y su traducción en relato les imprimen.

11 Agradezco a Max Hidalgo Nácher el envío a la lectura de El discurso de la servidumbre voluntaria de Etienne de la Boétie en diálogos previos al coloquio celebrado en Florianópolis.

12 Esta cuestión es abordada desde el humor producido a partir de una ingeniosa combinación del absurdo y del grotesco (Rinesi 2010:2): el comediante Diego Capusotto y su coguionista Pedro Saborido dan vida a «Robotril», un personaje que espeja, desde la pantomima irónica, el recurso a la medicación en función de volver soportable el régimen actual de «servidumbre voluntaria». La construcción solicita el modo en que transitamos nuestro presente: «la realidad es siempre alimentada por más elementos y relaciones que las que nuestro conocimiento supone», afirma Horacio González (13) a propósito de Peter Capusotto y sus videos. La densidad del humor de Capusotto se expresa, fundamentalmente, en la sutileza para interrogar este desconocimiento traducido en los modos de atravesar los «tiempos que corren» (Rinesi, 2010:2). Robotril replica una lengua que hablamos. Y es, justamente, la dimensión tragicómica la que provoca la identificación que «mueve a la risa» (González:12). Una risa transida por la imposibilidad de ir más allá de muy acotadas nano-intervenciones que poco pueden hacer frente al «desamparo propio de las marionetas» (13). Una risa provocada por un discurso ajeno al cinismo: posición de enunciación que explica, en parte, un efecto de lectura no exento de incomodidad y de molestia.

13 En Dufourmantelle el término "obsesión» tiene un sentido positivo en tanto da cuenta de una repetición apasionada que configura una marca del sujeto: «Un falsificador puede imitar el gesto del pintor o el estilo de un escritor, y hacer imperceptible su diferencia, pero jamás podrá hacer suya su obsesión» (1997:10).

14 En las grietas que se abren en el tejido social encontramos, junto a Eduardo Rinesi, la posibilidad para la acción política entendida como «la actividad o el conjunto de actividades desarrolladas en ese espacio de tensión que se abre entre las grietas de cualquier orden precisamente porque ningún orden agota en sí mismo todos sus sentidos ni satisface las expectativas que los distintos actores tienen sobre él» (2003:23).

15 En otros lugares he discutido la reducción del término «político» al plano declarativo y, más aún, partidario. a partir de un análisis de las políticas de la desconstrucción derrideana (cf. Gerbaudo 2007, 2014). Al respecto, este evento internacional es ilustrativo ya que reúne diferentes posiciones sobre la cuestión. Efectivamente, el 6 y 7 de diciembre de 2008 se celebró en París el Colloque International Derrida politique. Este coloquio (organizado por el Institut des Hautes Etudes en psychanalyse, el Laboratoire Disciplinaire «Pensées des sciences» [École Normale Supérieure], los archivos Husserl [ENS-CNRS] y el Comité editorial de la obra de Derrida con motivo de la aparición de Derrida pour les temps à venir y La bête et le souverain publicados respectivamente por las editoriales Stocky Galilée, también patrocinantes del evento) se difundía previamente con la siguiente introducción, incluida luego en el programa de mano: «Si la pensée derridienne ne développe pas une "philosophie politique" au sens traditionnel, c'est-à-dire toujours marquée par l'histoire de la métaphysique, elle ne constitue pas moins un mode de pensée fondamentalement politique. Mais autrement. Cette pensée demeure incomprise pour "ceux qui ne reconnaissent le politique qu'à l'aide des panneaux de signalisation d'avant la guerre". Toutes les interventions de Derrida dans des situations politiques concrètes s'inscrivent dans le prolongement d'un acte de déconstruction qui se situe en amont des habituelles prises de position qu'appellent les préjugés de la conscience bavarde. Leurs gesticulations tournent court devant la "chose même" du politique en ne cessant de répéter, sans même la conscience ou la mémoire du ressassement. S'il n'y a pas de démocratie sans mise en commun, il n'y a pas non plus de démocratie sans respect de la singularité et de l'altérité irréductible qui échappent à toute communauté. Dans l'urgence de penser ce qui vient de nouveau, de penser de manière neuve ce qui est nouveau et ce qui revient —en parlant de la politique sans parler politique-, Derrida nous invite à révolutionner le concept même de révolution». El programa anunciaba la lectura de «prises de position politique par Jacques Derrida rappelées par Chantal Talagrand et Daniel Mesguich» entre las exposiciones que, más allá de la apertura a cargo de Marc Crépon y René Major, pueden dividirse en dos grupos: las que se centran en cuestiones políticas ligadas especialmente al orden declarativo (cf. Bennington, Anidjar, Regazzoni, Kamuf, Major, Alunni, Lisse, Ukai, Mallet) y las que 
focalizan en performances con derivas políticas mayormente asociadas a su trabajo sobre la literatura y/o la escritura (cf. Michaud, Goldschmit, Dutoit, Cixous, Dufourmantelle, Habib, De Peretti y Rocha, Ronell, Nancy). Es oportuno mencionar que esta taxonomía es congruente con el resto de la producción de los conferencistas sobre la cuestión. Por ejemplo, la exposición de Cristina De Peretti y Delmiro Rocha, «Des ses premières textes», se articula recursivamente con los «primeros textos» que De Peretti escribía sobre Derrida, la desconstrucción y su trabajo político. En su muy temprano artículo «Las barricadas de la descontrucción» señalaba: «La desconstrucción (...) no opera nunca exclusivamente sobre enunciados, sobre contenidos de sentido, sobre la "edificación" formal y semántica de los filosofemas. Se ejerce asimismo sobre las relaciones y estructuras institucionales (políticas, económicas, sociales), sobre las formas históricas de su configuración» (42). Asimismo, cabe subrayar la elocuente condensación lograda en el título del ensayo de De Peretti y Rocha: un énfasis que marcha a contrapelo de la perspectiva dominante en el coloquio. Es decir, la pronunciada atención a sus "prises de position politique» y a textos como Spectres de Marx (1993) y La bête et le souverain (2008) no contribuyen a despejar los malentendidos en torno a la desconstrucción leída desde el «hilo» (cf. Derrida 1972b) escogido para aquella ocasión. Más aún: abonan la reducción de su trabajo político a sus aspectos explícitos y declarativos que además, según una más o menos extendida vulgata, comenzarían con Spectres de Marx para pronunciarse después. Al respecto cabría realizar una distinción, en la misma línea que la que plantean Nancy (2008), De Peretti y Rocha: ya en 1982, en una entrevista realizada por Christian Descamps, Derrida diferencia el posicionamiento radical exigido por situaciones que no admiten «desconstrucción» («Las críticas frontales y simples son necesarias siempre, son la ley de rigor en la urgencia moral o política, incluso si cabe discutir sobre la mejor formulación para este rigor. Tiene que ser frontal y simple la oposición a lo que pasa actualmente en Polonia o en Oriente Medio, en Afganistán, en El Salvador, en Chile o en Turquía, a las manifestaciones de racismo más próximas a nosotros, y a tantas cosas más singulares y sin denominación de Estado o Nación» —Derrida 1982:27—) del «pensamiento político» que se libra, «casi inaudible», a través de un trabajo sobre los «códigos» (29), sobre los protocolos institucionales. No olvidemos que ya en su poco convencional «defensa de tesis» despuntaba esta cuestión: «la forcé reproductive de l'autorité s'accommode plus facilement de déclarations ou de thèses soi-disant révolutionnaires dans leur contenu codé pourvu que soient respectés les rites de légitimation, la rhétorique et la symbolique institutionnelle qui désamorcent et neutralisent tout ce qui vient d'ailleurs. L'irrecevable, c'est ce qui, par-dessous les positions ou les thèses, vient de ranger ce contrat profond, l'ordre de ces normes, et qui le fait déjà dans la forme du travail, de l'enseignement ou de l'écriture» (1980b:451-451). Esta afirmación, lejos de reducirse al plano declarativo, se actúa en performances entre las que se destacan, entre muchas otras, el recorte del corpus para esa misma escena «doctoral».

16 He planteado en otro lugar (cf. 2013) la necesidad de ciertas precauciones toda vez que se usan los términos «concepto» $\mathrm{y}$ «teoría» para referir a las derivas de sus formulaciones (las comillas con que suelo rodear dichos términos son las señales de esas prevenciones epistemológicas).

17 En la introducción a su monumental biografía sobre Derrida, Benoît Peeters recoge diferentes comentarios que este había proferido respecto de su forma de preservar sus textos: «"Nunca perdí ni destruí nada. Hasta los papelitos (...) que Bourdieu o Balibar me dejaban en mi puerta (...), tengo todo. Las cosas más importantes y las cosas aparentemente más insignificantes» (Derrida en Peeters 2010:17). Paradójicamente, quien elabora una de las teorías más sofisticadas y prolíficas sobre el archivo dada la importancia que concede tanto a la selección del material a preservar como a su consignación en un lugar público y en soporte resistente (cf. Derrida 1995, 2003b), no podía evitar la tendencia a guardarlo todo: «La gran fantasía (...) es que todos esos papeles, libros o textos, o disquetes, ya me están sobreviviendo. Ya son testigos. Todo el tiempo pienso en eso, en quién irá —quién podría ir- después de mi muerte a mirar, por ejemplo, aquel libro que leí en 1953 y preguntarse: “¿Por qué tachó esto?, ¿por qué puso una flecha aquí? Estoy obsesionado por la estructura sobreviviente de cada uno de esos trozos de papel, de esas huellas"» (17). Estos dos pasajes son sólo muestras de la enorme tarea de Peeters al emprender su biografía: se trata no sólo, tal como Peeters contabiliza, de revisar sus ochenta obras publicadas sino también los innumerables textos y entrevistas no editados en forma de libro, los cursos y seminarios inéditos, los trabajos escolares y cuadernos personales, los manuscritos de sus libros, las transcripciones de entrevistas y mesas redondas, los artículos periodísticos y la correspondencia. Me permito comentar que si en 2005 presenté en la Universidad 
Nacional de Córdoba una tesis doctoral con la ilusión de haberlo hecho después de haber leído "toda la obra de Derrida», hoy advierto el carácter im-posible de esa empresa. Si Peeters sintió la necesidad de traducir la angustia y la soledad de sus decisiones ante la necesidad de procesar semejante cantidad de material vía un cuaderno de notas, Trois ans avec Derrida. Les carnets d'une biographe, personalmente creo productivo volver sobre la imagen derrideana de lectura desarrollada en los primeros párrafos de «La pharmacie de Platon» (1972b:71-72): de la enorme trama configurada por su producción, una trama im-posible de abarcar completa, sigo hasta donde puedo, con obsesión, un hilo. A partir de ese límite propongo, no obstante, mi lectura que no deja de abrigar la ahora sabida «fantasía» de «leer todo Derrida».

\section{Referencias bibliográficas}

Alunni, C. (2008). D'un autre cap. Colloque International Derrida politique. París: ENS.

Anidjar, G. (2008). Politiques de la laïcité. Colloque International Derrida politique. París: ENS.

Bennington, G. (2008). Politique, Derrida!. Colloque International Derrida politique. París: ENS.

Bourdieu, P. (1984). Homo academicus. París: Minuit.

(1985). Effet de champ et effet de corps. Actes de la Recherche en Sciences Sociales, (59), 2-73.

(1999). La domination masculine. París: Du Seuil.

(2001). Science de la science et reflexivité. Cours du Collège de France 2000-2001. París: Raisons

d'agir.

Butler, J. (1990 [1999]). El género en disputa. El feminismo y la subversión de la identidad. México: UNAM, 2001.

Cadava, E. y Connor, P. (1991). Preface. En E. Cadava, P. Connor y J. L. Nancy, (Eds.). Who comes after tthe Subject? NewYork: Routledge, vii.

Carles, P. (2001). La sociologie est un sport de combat. Francia: CP Productions y VF Films.

Cixous, H. (2008). Table ronde «Déconstruction/Psychanalyse du politique». Colloque International Derrida politique. París: ENS.

Compagnon, A. (1998). Le démon de la théorie. Littérature et sens commun. París: Du Seuil.

Chun, S. (2017). Política y ética en el pensamiento de Jacques Derrida: la hospitalidad incondicional como apertura hacia una «democracia por venir». Tesis doctoral. Buenos Aires: Universidad de Buenos Aires.

De Peretti, C. (1989). Las barricadas de la desconstrucción. Anthropos. Revista de Documentación Científica de la Cultura, (93), 40-43.

De Peretti, C. y D. Rocha (2008). Dès ses premières textes. Colloque International Derrida politique. París: ENS.

Derrida, J. (1967). De la grammatologie. París: Minuit.

- (1972a). signature événement contexte. En Marges de la philosophie. París: Minuit, 365-393. (1972b). La dissémination. París: Du Seuil.

(1974). Glas. París: Denoël/Gonthier, 1981.

(1980a). Envois. En La carte postal. De Socrate à Freud et au-delà. París: Flammarion, 5-273.

(1980b). Ponctuations: le temps de la thèse. En Du droit à la philosophie. París: Galilée, 1990, 439-461.

(1980c). La loi du genre. Parages (Nouvelle édition revue et augmentée). París: Galilée, 2003, $233-266$.

(1982). Jacques Derrida. Entrevista con Christian Descamps. Anthropos. Revista de Documentación Científica de la Cultura, 93, (1989), 26-29. 


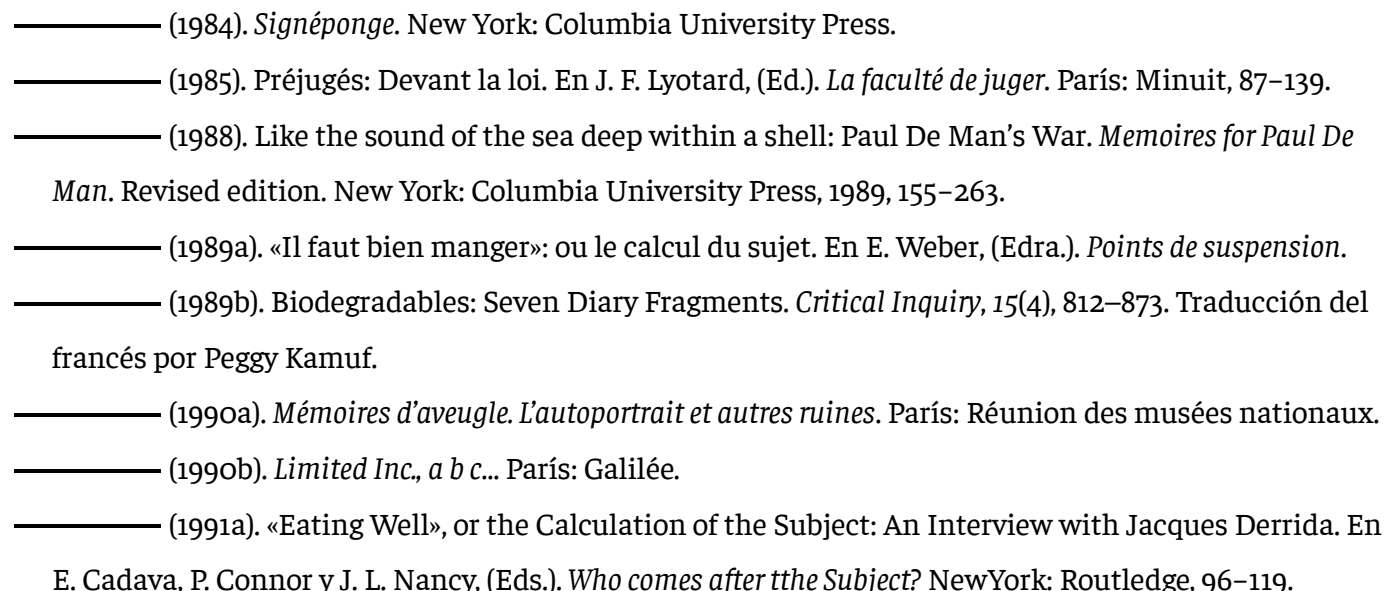

E. Cadava, P. Connor y J. L. Nancy, (Eds.). Who comes after tthe Subject? NewYork: Routledge, 96-119. - (1991b). Donner le temps. I. La fausse monnaie. París: Galilée. Traducción al español por Cristina

De Peretti.

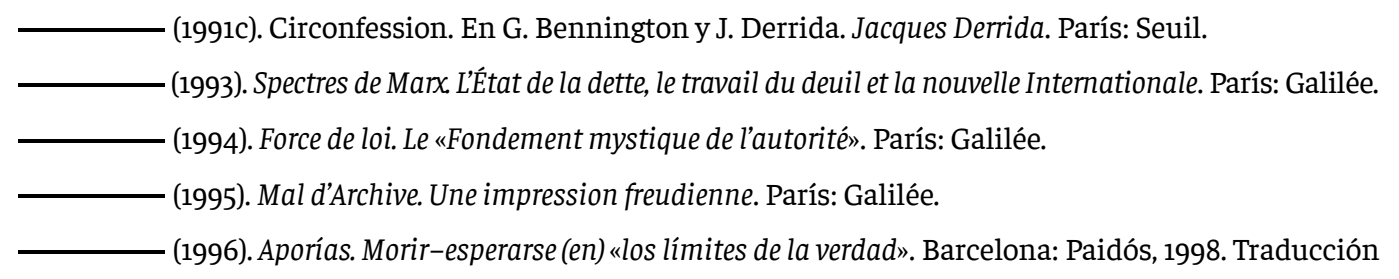
al español por Cristina De Peretti.

- (1997). Question de l'étranger: venue de l'étranger; Pas de hospitalité. En Anne Dufourmantelle invite Jacques Derrida à répondre De l'hospitalité. Calmann-Lévy: París, 11, 70; 71-137.

- (1998a). Un ver à soie. Points de vue piqués sur l'autre voile. Voiles. París: Galilée, 23-85.

- (1998b). Non pas l'utopie, l'im-possible. En Papier Machine. Le ruban de machine à écrire et autres réponses. París: Galilée, 2001, 349-366.

(1999). L'animal que donc je suis (À suivre). En M. Mallet, (Edra.). L'animal autobiographique. Autour de Jacques Derrida. París: Galilée, 251-301.

(2000). Lettres sur un aveugle. Punctum caecum. Tourner les mots. Au bord d’un film. París: Galilée, $71-126$.

- (2001a). Escoger su herencia. En J. Derrida y É. Roudinesco Y mañana qué... Buenos Aires: Fondo de Cultura Económica, 2002, 9-28. Traducción de Víctor Goldstein.

- (2001b). iPalabra! Instantáneas filosóficas. Madrid: Trotta. Traducción de Cristina de Peretti y Paco Vidarte.

(2001c). L’Université sans condition. París: Galilée.

(2003a). Auto-immunités, suicides réels et symboliques. Un dialogue avec Jacques Derrida; La déconstruction du concept du terrorisme selon Derrida. Le «concept» du 11 septembre. Dialogues à New York (octobre-décembre 2001). París: Galilée, 133-244.

-(2003b). Genèses, généalogies, genres et le génie. Les secrets de l'archive. París: Galilée.

(2008). Séminaire La bête et le souverain. Volume 1 (2001-2002). París: Galilée.

(2010). Séminaire La bête et le souverain. Volume 1 (2001-2002). París: Galilée.

Dufourmantelle, A. (1997). Invitation. En Anne Dufourmantelle invite Jacques Derrida à répondre De l'hospitalité. Calmann-Lévy: París, 10-136.

- (2006). American philo. Entretiens avec Avital Ronell. París: Stock.

(2008). Table ronde «Déconstruction/Psychanalyse du politique». Colloque International 
Derrida politique. París: ENS.

-(2011). Éloge du risque. Payot: 2014.

(2012). Intelligence du rêve. Fantasmes, apparitions, inspiration

(2014). Pour qui, pour quoi risquer ou donner sa vie aujourd'hui? En La Grande Table. France Culture. Emisión del 2 de junio.

-(2016). Au risque d'être soi. En Les Nouvelles Vagues. France Culture. Emisión del 18 de enero.

Dutoit, T. (2008). «Je suis une cigale»: 1" «Animot» poético-politique. Colloque International Derrida politique. París: ENS.

Eribon, D. (2018). L’héritage structuraliste. En France Culture. Emisión del 15 de agosto.

Foucault, M. (1971). Vigilary castigar. Nacimiento de la prisión. Buenos Aires: Siglo XXI, 1989. Traducción al español de Aurelio Garzón del Camino.

Freud, S. (1895). Estudios sobre la histeria. En Obras completas. Tomo 1. Barcelona: Losada, 1997, 39-168. Traducción de Luis López Ballesteros y De Torres.

Gasparri, J. (2016) Perspectivas sexogenéricas: literatura, artes y política. Badebec, (5/6). https://revista. badebec.org/index.php/badebec/issue/view/12

Gerbaudo, A. (2007). Derrida y la construcción de un nuevo canon crítico para las obras literarias. Córdoba: Universitas/Sarmiento/UNC.

(2013). Por esa forma de huelga llamada «desconstrucción» (o las insospechadas derivas de una cita). En M. Cragnolini, (Edra.). Entre Nietzschey Derrida. Buenos Aires: La cebra, 127-140.

-(2014). Algo más sobre protocolos de escritura, literatura y política en Jacques Derrida. Coloquio Homenaje a Jacques Derrida «La soberanía en cuestión». Buenos Aires: Museo del Libro y de la Lengua. -(2018). El fuego, el agua, la biodegradabilidad. Apuntes metodológicos para un archivo porvenir. En D. Vigna y P. Arán, (Comps.). Archivos, arte y medios digitales. Teoría y práctica. Córdoba: Centro de estudios avanzados, 41-65.

Goldschmit, M. (2008). La politique depuis la fin du monde. Colloque International Derrida politique. París: ENS.

González, H. (2010). A la sombra de Bombita Rodríguez. En R. Carbone y M. Murada, (Comps.). La sonrisa de mamá es como la de Perón. Los Polvorines: UNGS, 7-14.

Habib, S. (2008). Table ronde «Déconstruction/Psychanalyse du politique». Colloque International Derrida politique. París: ENS.

Héritier, F. (2012a). Le Sel de la vie. Lettre à un ami. París: Odile Jacob. (2012b). Entrevista con François Noudelmann. France culture.

(2017). Au gré des jours. París: Odile Jacob.

Hofstadter, Ds. (1979). Gödel, Escher, Bach. Un Eterno y Grácil Bucle. Barcelona: Tusquets, 1998. Traducción al español de Mario Usabiaga y Alejandro López Rousseau.

Kamuf, P. (2008). Le philosophe, en tant que tel, et la peine de mort. Colloque International Derrida politique. París: ENS.

Lacan, J. (1975-1976). El sinthome. Buenos Aires: Paidós, 2015. Traducción de Nora González.

Lisse, M. (2008). Une politique pour la vie. Colloque International Derrida politique. París: ENS.

Major, R. (2008). Table ronde «Déconstruction/Psychanalyse du politique». Colloque International Derrida politique. París: ENS.

Mallet, M. (2008). Le horla ou la folie de l'ipséité souveraine. Colloque International Derrida politique. París: ENS. 
Michaud, G. (2008). Seuils et tremblements. Colloque International Derrida politique. París: ENS.

Nancy, J. (2007). À plus d’un titre. Jacques Derrida. Sur un portrait de Valerio Adami. París: Galilée. (2008). Le secret, le sens. Colloque International Derrida politique. París: ENS.

Peeters, B. (2010). Derrida. París: Flammarion.

Regazzoni, S. (2008). Au-delà de la pulsion de pouvoir. Derrida et la déconstruction de la souveraineté. Colloque International Derrida politique. París: ENS.

Rinesi, E. (2003). Política y tragedia. Hamlet entre Hobbes y Maquiavelo. Buenos Aires: Colihue. (2010). Palabras liminares. En R. Carbone y M. Murada, compiladores. La sonrisa de mamá es como la de Perón. Los Polvorines: UNGS, 1-4.

Rodríguez Freire, R. (2018) La condición intelectual. Informe para una academia. Santiago de Chile: Mimesis. Ronell, A. (1992). Crack Wars: Literature, Addiction, Mania. Lincoln: University of Nebraska Press. (2000). Confessions of an Anacoluthon: Avital Ronell on Writing, Technology, Pedagogy, and Politics. Selected Works of Avital Ronell. Illinois: University of Illinois Press, 2008.

- (2002). Stupidity. Urbana: University of Illinois Press. (2003). The Experimental Disposition: Nietzsche's Discovery of America (Or, Why the Present Administration Sees Everything in Terms of a Test. American Literary History 15, (3), 560-74.

-(2006). American philo. Entretiens avec Anne Dufourmantelle. París: Stock. (2008). Derridémocratie. Colloque International. Derrida politique. París: ENS. (2011). Entretien. En V. Kauffman La faute à Mallarmé. L'aventure de la théorie littéraire. París: Du Seuil, 290-296.

(2012). Loser sons. Politics and authority. Illinois: University of Illinois Press.

Todorov, T. (2007). La littérature en péril. París: Flammarion.

Ukai, S. (2008). De «monstrueux comme si». Pour une historie du mensonge en politique au Japon. Colloque International Derrida politique. París: ENS.

Vidarte, P. (2006). Derriladacan: contigüidades sintomáticas. Sobre el objeto pequeñoj@cques. I Jornadas Internacionales Jacques Derrida. Buenos Aires: UBA

Zizek, S. (1992). ¡Goza tu síntoma! Jacques Lacan dentro y fuera de Hollywood. Buenos Aires: Nueva Visión, 1994. Traducción de Horacio Pons. 